\title{
EFFETS NÉGATIFS DE PH EXTRÊMES SUR LE DÉVELOPPEMENT EMBRYONNAIRE ET LARVAIRE DU BROCHET ESOX LUCIUS L.
}

\author{
H. LE LOUARN (1) et D.J. WEBB (2)
}

(1) Institut National de la Recherche Agronomique, Laboratoire d'Ecologie Aquatique, 65 rue de Saint-Brieuc, 35042 Rennes Cedex, France.

(2) Physiologie Cellulaire, Université de Rennes I, Campus de Beaulieu, avenue du Général Leclerc, 35042 Rennes Cedex, France.

\section{RÉSUMÉ}

Parmi les facteurs abiotiques conditionnant la survie au cours de la vie embryonnaire et larvaire des poissons, le pH semble jouer un rôle primordial. La motivation de ce travail provient d'observations sur les importantes variations de ses valeurs au printemps, l'existence de $\mathrm{pH}$ très acides en étangs forestiers et des mortalités importantes de brochetons au cours de la même période.

L'expérimentation s'est déroulée en aquariums avec une gamme de valeurs allant de 4,5 à 10,5 unités pH. Des tests en bains, continus ou momentanés (1 heure), sont effectués. Deux stades sont étudiés : les oeufs fécondés suivis jusqu'après l'éclosion ; les alevins incubés à pH neutre suivis jusqu'à la résorption de la vésicule vitelline.

Les paramètres suivants sont étudiés : survie, déroulement de l'embryogenèse, anomalies éventuelles, performances de nage. Le rythme cardiaque de l'embryon a été mesuré.

Les résultats sont les suivants :

- Les valeurs létales sont respectivement de 6 et 10 (alevins) et 4,5 et 10,5 (embryons).

- L'action sur les oeufs est moins forte, même si une mortalité importante $(46,7 \%)$ et de faibles performances de nage pour un $\mathrm{pH}$ inférieur à 6 semblent hypothéquer la réussite du recrutement. La valeur de 10,5 provoque un développement déficient des quelques individus survivants.

- Sur les alevins, même en bains d'une heure, la survie est faible : $42,6 \%$ à pH 5 , $29,3 \%$ à pH 10. Le rythme cardiaque ne variait pas de façon notable entre les seuils de tolérance.

Ces résultats permettent de récapituler pour une espèce largement distribuée les conditions de recrutement liées à l'acidité du milieu. II faut noter que les hausses brutales du $\mathrm{pH}$ au printemps dans des plans d'eau sur substrat cristallin rendent aléatoires la réussite des empoissonnements en brochetons des premiers stades.

Mots-clés : pH, oeufs, alevins, brochet, Esox lucius. 


\section{NEGATIVE EFFECTS OF EXTREME ENVIRONMENTAL PH ON EMBRYONIC AND LARVAL DEVELOPMENT OF THE PIKE ESOX LUCIUS L.}

\section{ABSTRACT}

The survival of fish embryos and larvae depends on many environmental factors and $\mathrm{pH}$ is of particular importance. The present study was investigated following the observation that, during the same period in spring, the $\mathrm{pH}$ was extremely basic in same ponds or very acid in forest ones, and the mortality of young pike very high.

Experiments were carried out in aquariums with a range of $\mathrm{pH}$ from 4.5 to 10.5 . Incubation at a particular value was either continuous or of short duration ( $1 \mathrm{~h}$ ). Two developmental stages were studied : fertilized eggs up to hatching ; alevins followed until absorption of the yolk sac.

The parameters analysed were the following : survival, development, abnormalities, swimming performance and heart rate.

The following results were obtained:

- The lethal $\mathrm{pH}$ values were 6 and 10 for alevins, 4.5 and 10.5 for unhatched embryos. The unhatched embryos were less sensitive, although a high mortality $(46.7 \%)$ and weak swimming performance at $\mathrm{pH}$ below 6 would reduce the success of recruitment. The few survivors at $\mathrm{pH} 10.5$ showed abnormal development.

- For alevins, even a short exposure of 1 hour reduced survival considerably at pH 5 $(42.6 \%)$ and $\mathrm{pH} 10(29.3 \%)$. There was a noticeable effect on heart rate into the tolerated $\mathrm{pH}$ range.

These results allow a reconsideration of the conditions for recruitment of a widely distributed species according to the $\mathrm{pH}$. In particular the sharp increases in $\mathrm{pH}$ in spring of the soft water ponds make the stocking with early stage pike much more hazardous.

Key-words : $\mathrm{pH}$, eggs, alevins, pike, Esox lucius.

\section{INTRODUCTION}

Le $\mathrm{pH}$ est l'un des principaux facteurs abiotiques conditionnant la survie des populations de poissons d'eau douce. L'action néfaste des valeurs très faibles sur le peuplement des eaux intérieures fut signalée par VALLIN (1952) et attribuée à l'augmentation du $\mathrm{SO}_{2}$ dans l'atmosphère, mais dès le début du siècle les effets de l'acidification des lacs étaient soupçonnés (MILBRINK et JOHANSSON, 1975). Les premières études furent menées sur le vairon du Canada Pimephales promelas (MOUNT, 1973), le poisson-zèbre Brachydanio rerio et le brochet Esox lucius (JOHANSSON et KIHLSTRÖM, 1975).

Suivant les auteurs, la valeur létale se situerait vers 4,5-5 (SCHÄPERCLAUS, 1962), 5 (ALABASTER et LLOYD, 1980), voire moins en cas d'acclimatation (RYNANEN, 1961, in ALABASTER et LLOYD, 1980). Cependant, ALMER (1972, in JOHANSSON et KIHLSTRÖM, 1975) note un dysfonctionnement du recrutement de certaines espèces pour des $\mathrm{pH}$ plus élevés, indiquant une sensibilité plus grande des premiers stades. JOHANSSON et KIHLSTRÖM (1975) confirment ces conclusions en contradiction avec les 
conclusions d'une revue de l'E.I.F.A.C.(1969) qui fixent à 4-4,5 la valeur du pH permettant la reproduction du brochet.

Beaucoup moins d'observations traitent de l'influence des pH élevés. Dans les régions à substrat pauvre en calcaire, les variations en sont pourtant fortes, en liaison avec le développement du phytoplancton et des macrophytes dans les milieux eutrophes. Des valeurs supérieures à 10 sont fréquentes, comme dans deux étangs expérimentaux (Figure 1) où la survie d'alevins a été très faible.

Pour ALABASTER et LLOYD (1980), les premières influences négatives sur les jeunes stades se produiraient pour des valeurs de 9,5-10 et le brochet ne supporterait pas une exposition prolongée à des valeurs supérieures.

L'étude présente s'intègre dans une revue des causes de mortalité précoce du brochet. Menée à la station expérimentale INRA du Rheu, elle est motivée par des observations de très faibles survies après déversements expérimentaux d'oeufs embryonnés ou d'alevins vésiculés de brochet dans des milieux à pH extrêmes : étangs de type forestier à pH très acides (5 à 6 ), plans d'eau eutrophes atteignant au cours du printemps des valeurs de 10 à 11 .

\section{MATÉRIEL ET MÉTHODES}

\section{Gamme de pH}

Les valeurs utilisées sont celles observées dans les milieux suivis pour la reproduction et le développement précoce du brochet, comprises entre 5 et 10,5 (Figure 1).

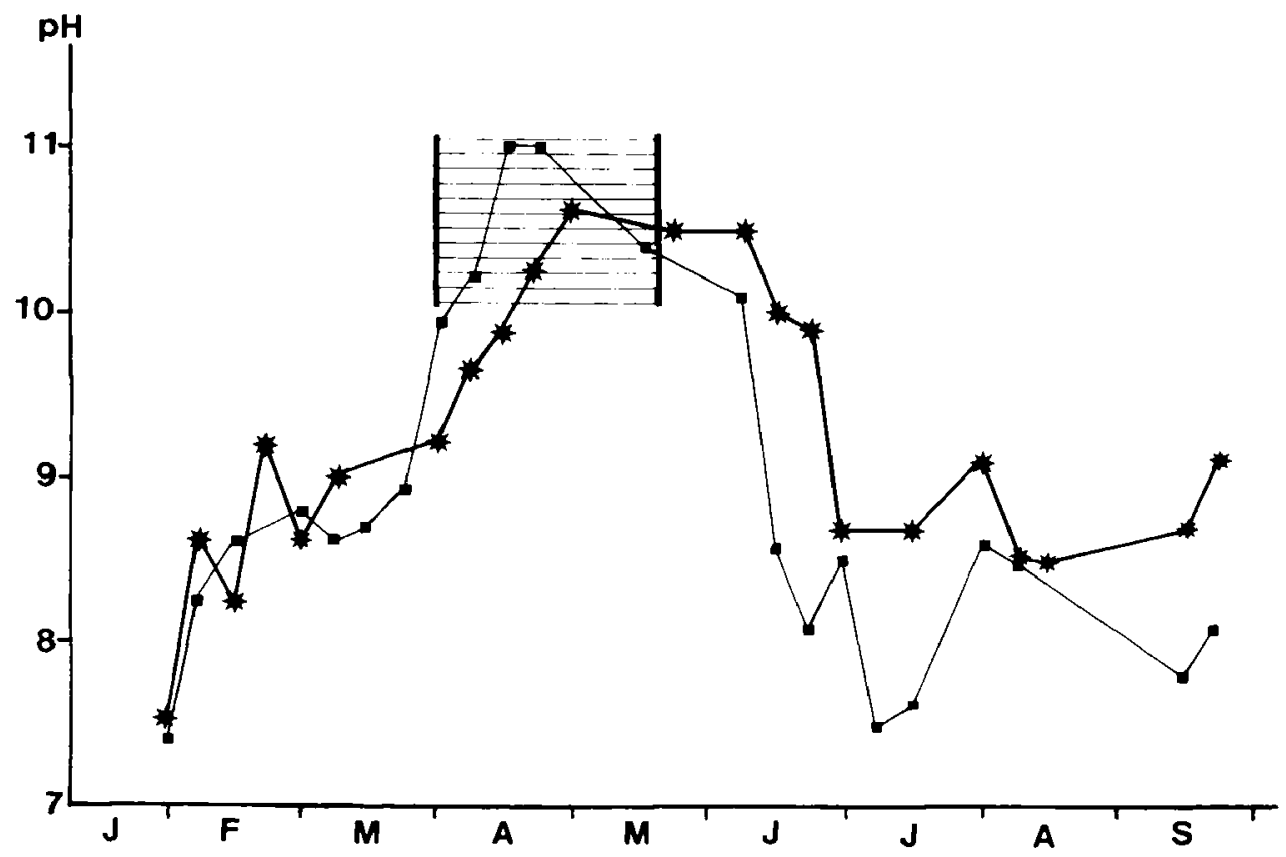

Figure 1

Valeurs moyennes hebdomadaires des $\mathrm{pH}$ dans deux étangs expérimentaux de la station expérimentale. Période de développement embryonnaire et larvaire du brochet.

\section{Figure 1}

Mean weekly values of the $\mathrm{pH}$ in two experimental ponds. Pike embryonic and larval period. 
Deux problèmes ont été rencontrés lors de la préparation des bains de référence :

- Les solutions tampons préconisées par BRUNEL (1948) et DIEM et LENTNER (1972), nitrate-phosphate-borate/ $\mathrm{HCl}$ pour les valeurs faibles, phosphate monopotassiquephosphate disodique pour les valeurs fortes, entraînent des conductivités proches de $1000 \mu \mathrm{S} / \mathrm{cm}$ donc des risques réels de perturbation pour le matériel animal (CHULAKASEM et al., 1989).

Des essais effectués avec des fixateurs de pH utilisés en aquariophilie, ou après circulation sur de la tourbe, n'ont pas évité de fortes variations de ce paramètre en quelques heures d'observation.

- Les solutions sont donc préparées à partir d'eau provenant, soit de mares forestières pour les $\mathrm{pH}$ acides, soit de bassins eutrophisés pour les $\mathrm{pH}$ basiques. Les valeurs sont éventuellement ajustées par mélange avec une eau " neutre ". En changeant les solutions chaque jour, on évite des variations supérieures à une demi-unité sauf pour la valeur la plus basse. Les valeurs extrêmes de conductivité sont de 350 et $500 \mu \mathrm{s} / \mathrm{cm}$.

\section{Protocole d'étude}

Les effets éventuels d'une balnéation momentanée sont intéressants à noter pour juger du type d'action du paramètre étudié (immédiat ou à plus long terme). De plus, en milieu de journée au printemps, une forte activité photosynthétique provoque dans des étangs eutrophes une augmentation temporaire du $\mathrm{pH}$. C'est pourquoi deux types de balnéation, continue et momentanée en début d'expérience, sont étudiés.

Toutes les manipulations sont effectuées dans des aquariums de 20 litres, en circuit fermé. Les manipulations sont réduites au minimum sans aucun transport. L'effet du passage d'un aquarium à l'autre lors des balnéations d'une heure peut être considéré comme négligeable. Des tests (non publiés), portant sur la résistance à la dessiccation des oeufs de brochet, montrent en effet l'innocuité d'une émersion de plusieurs dizaines de minutes. Pour le test portant sur les alevins, des témoins ont subi la même manipulation, sans aucune conséquence. Les comptages en cours d'expérience s'effectuent sans émersion des oeufs ou alevins.

Pour chaque manipulation, les individus proviennent d'une même fratrie (même carafe de fécondation ou auge d'alevinage). Le pH du milieu d'origine était stable à 8,3. L'expérience s'est déroulée en écloserie à une température de $12^{\circ} \mathrm{C}$ et à la photopériode normale. Elle a été menée en deux phases :

- Des oeufs fécondés sont suivis de 35 degrés-jours à l'éclosion. Les survivants sont ensuite placés dans un milieu à pH neutre jusqu'à la fin de la résorption de la vésicule vitelline. La poursuite de l'étude aurait nécessité la fourniture de proies vivantes, elles-mêmes sous l'influence éventuelle du $\mathrm{pH}$ pour leur survie et/ou leur croissance. Trois réplicats de 20 oeufs sont placés dans des cagettes de grillage à maille de $0,5 \mathrm{~mm}$.

Le $\mathrm{pH}$ est contrôlé tous les jours et le milieu éventuellement changé. Les observations ont porté sur le déroulement et la durée de l'incubation, les mortalités journalières éventuelles, la survie finale, l'état des alevins à l'éclosion.

Les perturbations dans le comportement des organismes aquatiques, entre autres les performances de nage, sont utilisées comme indicateurs sensibles et rapides d'un stress toxique (HE et al., 1994). Ils ont été utilisés avec succès chez le poisson (LITTLE et FINGER, 1990). L'alevin de brochet durant sa préparation à la vie active est fixé à un support et passe par de courtes périodes de nage active avant un nouveau repos. Une 
déficience dans son développement l'amène à se rapprocher de plus en plus du fond. II est donc possible de mettre en relation les Hauteurs Moyennes de Fixation (H.M.F.) avec l'état général de l'alevin. BRY (comm. pers.) utilise ce test pour des études de l'effet de xénobiotiques sur le brocheton. La technique est décrite par AMBLARD (1996).

Dans l'expérimentation présente, chaque lot est placé dans un récipient gradué d'une hauteur totale de $20 \mathrm{~cm}$ dans une eau à $\mathrm{pH}$ neutre. Les mesures sont effectuées 4 fois par jour pendant les 4 premiers jours car la résorption de la vésicule vitelline s'accompagne ensuite de séquences de mouvements de plus en plus rapprochées.

- Des alevins dont l'incubation s'est déroulée à pH neutre, sont placés à l'âge de 3 jours et au stade de développement de 36 degrés-jours dans des milieux à pH variés et suivis jusqu'après l'éclosion. Trois réplicats de 50 alevins ont été utilisés. Les contrôles ont été effectués deux fois par jour et les comptages à $12 \mathrm{~h}, 24 \mathrm{~h}, 48 \mathrm{~h}$ et 120 heures. L'expérience s'est terminée avant la résorption complète de la vésicule vitelline pour éviter de comptabiliser des mortalités dues à un déficit alimentaire éventuel.

Parallèlement à cette expérimentation, le même matériel animal est utilisé dans le cadre d'une étude plus approfondie des besoins énergétiques au cours du développement en comparaison avec le medaka Oryzias latipes suivant deux protocoles :

- la mesure de la consommation d'oxygène par l'embryon. Des ajustements sont nécessaires avant l'utilisation des données,

- la mesure du rythme cardiaque de l'alevin (nombre de battements en 20 secondes). 20 alevins sont suivis du troisième au neuvième jour après éclosion, en solutions de $\mathrm{pH}$ de 5 à 10 , dans trois milieux : eau d'étang, liquide physiologique normal ou dilué au dixième, à $14^{\circ} \pm 1^{\circ} \mathrm{C}$. Les valeurs de $\mathrm{pH}$ sont fixées par différents tampons : MES pour les valeurs 5-6 ; HEPES pour 7,5; AMPSO pour 9,0 ; CAPSO pour 9,5-10. Le pH est contrôlé tous les jours et éventuellement ajusté. Pour les observations, 10 alevins sont transférés dans une boîte de Pétri et les battements cardiaques chronométrés par observation sous loupe binoculaire. Les résultats doivent être considérés comme préliminaires à une étude plus approfondie.

\section{RÉSULTATS}

\section{Phase 1 - Embryons exposés (Tableau I)}

On observe une mortalité totale pour une valeur de 4,5. Elle se produit précocement, au moment de la formation de la blastula et aucun développement de l'embryon n'est visible.

A pH 5,5 la survie est à l'éclosion de $63,3 \%$, mais une certaine mortalité se produit dans les 24 heures. A pH 6, la survie est de 66,6\%. Pour ces deux valeurs, le test de l'écartréduit indique une différence significative de $3 \%$ et $7 \%$ respectivement pour les survies en balnéations continue et momentanée, et le témoin. On note également une différence significative entre les résultats à $\mathrm{pH} 5,5$ en balnéations continue et momentanée $(p=0,03)$.

Entre les valeurs 7 et 9,5, aucune différence significative ne peut être notée.

$\mathrm{A} \mathrm{pH} \mathrm{10,5} \mathrm{la} \mathrm{survie} \mathrm{à} \mathrm{l'éclosion} \mathrm{est} \mathrm{de} \mathrm{3,3 \% .} \mathrm{On} \mathrm{n'a} \mathrm{pu} \mathrm{noter} \mathrm{que} \mathrm{la} \mathrm{formation} \mathrm{de}$ seulement 4 embryons. L'un des deux alevins éclos est mort le premier jour et l'autre présentait une malformation de la colonne vertébrale, le rendant non viable. L'éclosion s'est produite avec 24 heures d'avance sur celle des autres lots. 


\section{Tableau I}

Survie (\%) des oeufs incubés à différents $\mathrm{pH}(\mathrm{C})$ ou plongés pendant une heure dans les mêmes solutions (M). * : Différences significatives.

Table I

Survival (\%) of eggs incubated at different pH (C) or placed for one hour in the same solutions $(M)$. $*$ : Significant differences.

\begin{tabular}{|c|c|c|c|c|}
\hline $\mathrm{pH}$ & Lots & Survile \% & Ecarts types & Remarques \\
\hline \multirow[t]{2}{*}{4.5} & $\mathrm{C}$ & 0 & & Pas d'embryonnement \\
\hline & M & 80,0 & 8.16 & \\
\hline \multirow[t]{2}{*}{5,5} & C & 63.3 & $19.997]^{*}$ & Apres eclosian : survie $53.3(16.9)$ \\
\hline & $M$ & 83.3 & 4.71 & \\
\hline \multirow[t]{2}{*}{6} & $\mathrm{C}$ & 66.6 & 9.42 & \\
\hline & M & 80.0 & 8.16 & \\
\hline 7 Témoin & & 93,3 & $4.71 J$ & \\
\hline \multirow[t]{2}{*}{8} & C & 96.6 & 4.71 & \\
\hline & M & 96.6 & 4.71 & \\
\hline \multirow[t]{2}{*}{9.5} & c & 86.6 & 4.71 & \\
\hline & $M$ & 90,0 & 0 & \\
\hline \multirow[t]{2}{*}{10.5} & $\mathrm{C}$ & 3.3 & 4.71 & 4 embryons, 2 éclos dont 1 anorma \\
\hline & $M$ & 93.3 & 4,71 & \\
\hline
\end{tabular}

Les performances de nage des alevins éclos sont médiocres pour les faibles valeurs de $\mathrm{pH}$ (Tableau II). La signification des résultats est testée par deux analyses de variance. La première réalisée avec le modèle complet (toutes les variables et leurs interactions) montre que trois variables ont un effet significatif : le $\mathrm{pH}$, le jour et l'interaction jour-heure $(\mathrm{p}<0,0001)$. L'effet moyen de chaque valeur de $\mathrm{pH}$ est estimé après élimination des effets des covariances " jour " et " heure du jour ", et un test de Student permet de déterminer les valeurs dont les effets sont significativement différents. Toutes les situations, sauf une balnéation à pH 9,5 continue, apparaissent comme significativement différentes du témoin. Les résultats montrent une très nette réduction des performances des alevins ayant connu continûment ou non un environnement acide $(\mathrm{pH}=5,5)$. Pour des valeurs de $\mathrm{pH}$ légèrement acides $(\mathrm{pH}=6)$ ou basiques $(\mathrm{pH}=9,5)$, les performances se rapprochent de celles $\mathrm{du}$ témoin.

\section{Tableau II}

Hauteurs Moyennes de Fixation (en $\mathrm{cm}$ ) des alevins incubés à différents $\mathrm{pH}(\mathrm{C})$ ou placés dans les mêmes solutions pendant une heure $(M) . *: p<0,05 ; * *: p<0,01$.

\section{Table II}

Mean fixation heights (in $\mathrm{cm}$ ) of alevins incubated at different $\mathrm{pH}(\mathrm{C})$ or placed in the same solutions for one hour (M). $*: \mathbf{p}<0.05 ; * *: \mathbf{p}<0.01$.

\begin{tabular}{|c|c|c|c|c|c|c|c|}
\hline & \multicolumn{7}{|c|}{ LOTS } \\
\hline & $5,5 \mathrm{C}$ & 5,5 M & $6 \mathrm{C}$ & $6 \mathrm{M}$ & Témoin 7 & $9,5 \mathrm{C}$ & $9,5 \mathrm{M}$ \\
\hline J1 à J4 & $\begin{array}{c}1,17 \\
(0,15)\end{array}$ & $\begin{array}{c}1,25 \\
(0,68)\end{array}$ & $\begin{array}{c}1,22 \\
(0,46)\end{array}$ & $\begin{array}{c}1,43 \\
(0,35)\end{array}$ & $\begin{array}{c}2,96 \\
(0,63)\end{array}$ & $\begin{array}{c}1,80 \\
(0,69)\end{array}$ & $\begin{array}{r}1,33 \\
(0,38)\end{array}$ \\
\hline & $* *$ & $* *$ & $* *$ & $*$ & & & $* *$ \\
\hline $\mathrm{J3}$ et J4 & $\begin{array}{c}2,91 \\
(1,63)\end{array}$ & $\begin{array}{c}3,45 \\
(1,24)\end{array}$ & $\begin{array}{c}3,49 \\
(1,77)\end{array}$ & $\begin{array}{c}2,59 \\
(0,65)\end{array}$ & $\begin{array}{c}3,60 \\
(0,59)\end{array}$ & $\begin{array}{c}4,25 \\
(2,26)\end{array}$ & $\begin{array}{r}3,71 \\
(2,81)\end{array}$ \\
\hline
\end{tabular}


L'analyse ne laisse pas apparaître deux nettes différences entre une détérioration de l'environnement passagère ou continue. Néanmoins, les performances semblent être légèrement moins bonnes dans le second cas.

Les mesures effectuées durant les deux derniers jours (Tableau II) montrent pour les alevins survivants des performances de nage comparables à celles du lot témoin.

\section{Phase 2 - Alevins exposés}

Les alevins montrent une bonne survie entre les $\mathrm{pH} 7$ et 9 . En deça et au-delà, la mortalité est totale et intervient en moins de 24 heures, voire en moins de 12 heures pour la valeur de 10,5 (Figure 2).
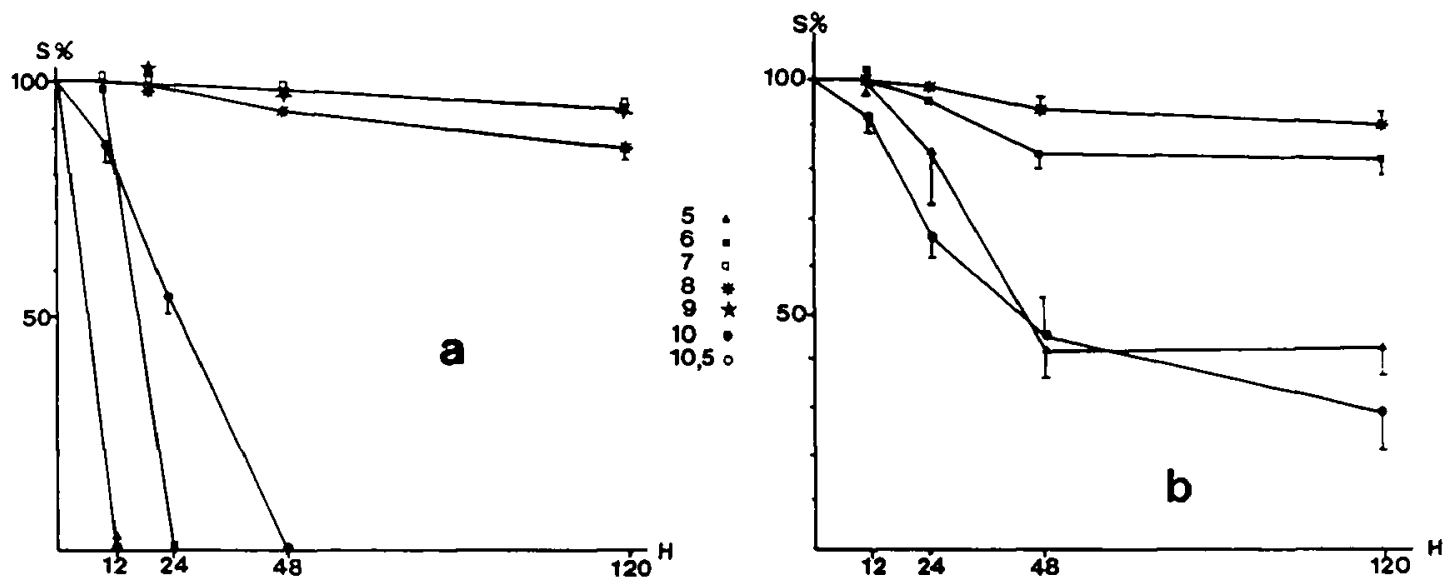

Figure 2

Survie (S\%) des alevins de 3 jours exposés à différents pH pendant $120 \mathrm{~h}$ (A) ou pendant la première heure d'expérience (B).

\section{Figure 2}

Survival (S\%) of 3-day-old alevins exposed at different $\mathrm{pH}$ for $120 \mathrm{~h}$ (A) or during the first hour (B).

En bains momentanés, la survie n'est différente de celle des témoins que pour les valeurs extrêmes : $(43,3 \%$ à $\mathrm{pH} 4,5,30 \%$ à pH 10$)$.

Le rythme cardiaque des alevins suivi du 3è jour à l'éclosion permet de confirmer certaines observations précédentes. Les effets les plus significatifs se notent pour les pH élevés. Avec les solutions provenant du milieu naturel régulées par différents tampons, on observe une mortalité totale en 6 à 9 jours avec un ralentissement du rythme cardiaque, après le 2 è jour à pH 10 (Figure 3). En solution de Yamamoto-Ringer seule la valeur de 10 est létale, tandis qu'en milieu dilué au dixième les résultats sont intermédiaires mais aussi plus irréguliers.

\section{DISCUSSION}

Les valeurs extrêmes du pH peuvent agir sur l'équilibre du milieu, la ressource en nourriture, le comportement à moyen ou plus long terme des poissons (ALABASTER et LLOYD, 1980 ; SCHRECK et MOYLE, 1990 ; BEKLIOGLU et MOSS, 1995 ; inter alia). L'étude présente se limite aux effets plus immédiats et plus directs sur les premiers stades de développement du brochet avant le début du nourrissage exogène. 

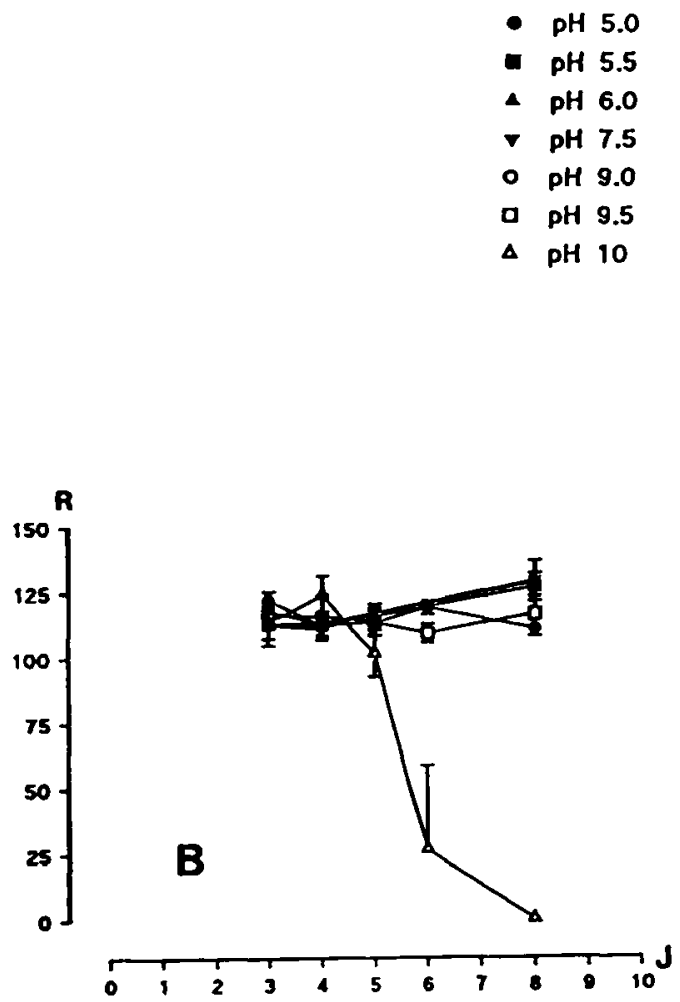

\section{Figure 3}

Rythme cardiaque ( $R$ en battements/minute) du 3è au 9è jour $(J)$ après éclosion des alevins en solutions de différents $\mathrm{pH}$. A : en eau provenant d'étangs. B en liquide physiologique Yamamoto-Ringer. $C$ en liquide physiologique au 1/10è.

\section{Figure 3}

Heart rate ( $R$ in beats/min.) of the alevins in solutions of different $\mathrm{pH}$, from 3 to 9 days $(J)$ after hatching. $A$ : in pond water. $B$ : in physiological solutions. $C$ : in diluted physiological solutions.

L'influence des $\mathrm{pH}$ sublétaux peut être mesurée par la perturbation des comportements alimentaires et les déficits de croissance qui en découlent (JOHANSSON et KIHLSTRÖM, 1975 ; CLEVELAND et al., 1988 ; THOMSEN et al., 1988). Pour le brocheton qui n'accepte que les proies vivantes, pouvant être elles-mêmes perturbées par les variations du milieu, il est indispensable d'étudier des manifestations plus immédiates. La consommation d'oxygène par l'embryon et le rythme cardiaque en font partie. La mesure de la consommation d'oxygène a posé des problèmes liés à la pureté du milieu d'observation. Les battements du coeur sont par contre très bien observables dès le troisième jour après la fécondation. Après l'éclosion, et avant le stade de nourrissage exogène, il a semblé pratique de mesurer le comportement typique du brocheton alternant périodes de fixation par papilles adhésives et périodes de nage active. La possibilité de s'élever dans la colonne d'eau rend bien compte de l'état physique de l'alevin. Le résultat est facilement mesurable. Par contre, le test ne peut durer que quelques jours.

Faute de pouvoir comparer les conditions d'étude, partois de simples observations en milieu naturel, des revues comme celles d'ALABASTER et LLOYD (1980) et SAYER et al. (1993) ne peuvent servir de référence sauf pour les valeurs extrêmes de 3,5 et 11 pouvant 
être considérées comme létales pour toutes les espèces. En fait, on ne peut définir une gamme de valeurs où un bon développement de l'individu et de la population est possible et un domaine où il ne l'est plus. II y a détérioration graduelle lorsque le $\mathrm{pH}$ s'éloigne des conditions moyennes.

Ainsi, des valeurs non létales pour l'ensemble entraînent des anomalies du développement accompagnant une mortalité plus ou moins marquée. La malformation de la colonne vertébrale observée à $\mathrm{pH}$ 10,5 est à rapprocher des observations de MILBRINK et JOHANSSON (1975) sur le gardon Rutilus rutilus à $\mathrm{pH}$ acide, à cause d'un défaut de calcification (READER et al., 1989 ; SAYER et al., 1993). L'éclosion précoce pour la même valeur confirme les résultats de TROJNAR (1977) obtenus chez le saumon de fontaine Salvelinus fontinalis. Chez la perche Perca fluviatilis, les deux phénomènes sont liés, comme dans l'expérience présente (RUNN et al., 1977, in TROJNAR, 1977).

\section{- Les effets directs des pH extrêmes sont de deux types :}

- on observe une précipitation du mucus sur l'épithélium branchial et des protéines à l'intérieur des cellules épithéliales. Ces réponses ont été notées pour les $\mathrm{pH}$ très acides (ELLIS, 1937 et WESTFALL, 1945, in ALABASTER et LLOYD, 1980 ; LLOYD et JORDAN, 1964, in DAYE et GARSIDE, 1980 ; DAYE et GARSIDE, 1976) et font obstacle à l'osmorégulation (LEIVESTAD et MUNIZ, 1976, in RASK, 1983). Les conséquences en sont probablement une mortalité rapide. Cette manifestation s'accompagne de blessures aux cellules épidermiques (externes, des branchies et des organes internes) ainsi qu'au foie, au cerveau et aux cristallins. Les effets sont les mêmes pour les $\mathrm{pH}$ très faibles et très forts, moins accentués pour ces derniers (DAYE et GARSIDE, 1980). Les conséquences en sont moins immédiates et une guérison est possible pour un certain nombre d'individus. Les résultats obtenus dans l'expérience présente par trempages momentanés semblent répondre à ce schéma et sont à rapprocher des conclusions de JOHANSSON et KIHLSTRÖM (1975) et MILBRINK et JOHANSSON (1975) sur l'effet des pH acides sublétaux. De même, à partir du troisième jour après éclosion les performances de nage des alevins survivants des différents lots sont comparables et aucune différence significative ne peut plus être notée (Tableau II),

- le principal effet indirect concerne l'influence de la concentration des ions hydrogène sur les formes ionisées ou non ionisées de certaines substances toxiques. Les formes non ionisées diffusent plus facilement à travers les membranes cellulaires. $\mathrm{Or}$, à $\mathrm{pH} 9, \mathrm{NH} 3$ constitue 35 à $45 \%$ de l'ammonium total d'un milieu contre $1 \%$ à pH 7 (SCHRECK et MOYLE, 1990). Ceci pourrait expliquer les meilleures survies des alevins de brochet maintenus dans les solutions de liquide physiologique.

Dans tous les cas, les jeunes stades sont les plus sensibles (MILBRINK et JOHANSSON, 1975 ; MENENDEZ, 1976 ; KWAIN et ROSE, 1985 ; SAYER et al., 1993). Par exemple, les adultes de tanche survivent plusieurs semaines à $\mathrm{pH}$ très acide malgré une hyperglycémie et une diminution du pH sanguin (DEMAËL et al., 1984).

Dans l'expérience présente, les oeufs ont résisté à des conditions plus drastiques, même si la survie des alevins après l'éclosion peut être compromise (mauvaises performances de nage). Une certaine protection est assurée par l'enveloppe chorionique (LEE et GERKING, 1980 ; RASK, 1984 ; SAYER et al., 1993), mais la barrière principale contre le passage des ions hydrogène et l'influence du milieu sur le $\mathrm{pH}$ intracellulaire $(\mathrm{pHi})$ est la membrane plasmique. De telles études sont très limitées chez les poissons. Cependant chez l'amphibien Xenopus laevis, il a été montré pour l'oeuf non fécondé que le $\mathrm{pHi}$ ne varie pas quand le $\mathrm{pH}$ du milieu fluctue entre 5 et 10 (WEBB et NUCCITELLI, 1982). Pour une espèce de poisson, le médaka Oryzias latipes, la conductance membranaire de l'oeuf non fécondé a été modifiée par une augmentation du pH du milieu (WEBB, 1991). Cet effet disparaît après la fécondation. 


\section{CONCLUSION}

Les résultats de cette étude, en accord avec ceux de GILLET et ROUBAUD (1986) sur les conditions de fécondation, permettent de récapituler les possibilités de survie liées à la teneur en ions hydrogène d'une espèce à vaste répartition géographique. II faut toutefois envisager une acclimatation possible, au moins aux $\mathrm{pH}$ acides : dans les pays scandinaves sont notées, quelle que soit l'espèce étudiée, des survies à des valeurs particulièrement faibles (Mc WILLIAMS, 1982).

D'un point de vue pratique, on doit se poser la question du devenir des déversements de brochets sous forme d'oeufs embryonnés ou d'alevins vésiculés dans des eaux à fort risque d'eutrophisation durant la période de développement précoce.

\section{REMERCIEMENTS}

Nous remercions pour leur aide Jacques PROU, responsable de la Pisciculture fédérale du Boulet, et Manuel PLANTEGENEST, statisticien à l'ENSAR.

\section{BIBLIOGRAPHIE}

ALABASTER J.S., LLOYD R., 1980. Water quality criteria for freshwater fish. FAO, Batterwoth ed., London, $297 \mathrm{p}$.

ALMER B., 1972. Försurningens inverkan pa fiskbestand i västkustsjöar. Inform. from Sötvattenslab. Drottningholm, 12, 1-47.

AMBLARD G., 1996. Eléments de caractérisation biologique d'un ensemble de mésocosmes aquatiques et de l'effet d'un insecticide carbomate, le carbofuran, sur les performances de jeunes brochets (Esox lucius). Mémoire de D.E.A, Toxicologie de l'Environnement. Université de Metz-Rouen-Strasbourg, $35 \mathrm{p}$.

BEKLIOGLU M., MOSS B., 1995. The impact of $\mathrm{pH}$ on interaction among phytoplankton algae, zooplankton and perch (Perca fluviatilis) in a shallow, fertile lake. Freshw. Biol., 33, 47-509.

BRUNEL A., 1948. Traité pratique de chimie végétale. Frère éd., Tourcoing, $412 \mathrm{p}$.

CHULAKASEM W., NELSON J.A., MAGNUSSON J.J., 1989. Interaction between effects of low ion concentration on mortality during early development of medaka Oryzias latipes. Can. J. Zool., 67, 2158-2168.

CLEVELAND L., INGESOLL C.G., BUCKLER D.R., 1988. Effects of simulated episodic pH depressions and aluminium on brook trout. 8. Ann. Int. Symposium on Lake and Waters Man., page 6.

DAYE P.G., GARSIDE E.T., 1976. Histopathologic changes in surficial tissues of brook trout, Salvelinus fontinalis (Mitchill). Can. J. Zool., 53, 639-641.

DAYE P.G., GARSIDE E.T., 1980. Development, survival, and structural alterations of embryos and alevins of Atlantic salmon, Salmo salar L., continuously exposed to alcaline levels of $\mathrm{pH}$, from fertilization. Can. J. Zool, 58, 369-377.

DEMAËL A., GUSTIN P., LEPOT D., 1984. Influence d'une baisse modérée du pH de l'eau sur quelques activités enzymatiques du foie et sur certains composants sanguins de la tanche (Tinca tinca L.). Ichtyoph. Acta, 8, 75-91.

DIEM K., LENTNER C., 1972. Tables scientifiques. Doc-geigy, Ciba-geigy eds, Bâle, 863 p.

E.I.F.A.C., 1969. Water quality criteria for European freshwater fish. Extreme pH values. Water Res., 3, 593-611.

ELLIS H.M., 1937. Detection and measurement of stream pollution. Bull. U.S. Dep. Commer., 27, 
GILLET C., ROUBAUD P., 1986. Survie embryonnaire précoce de 9 espèces de poissons d'eau douce après un choc de $\mathrm{pH}$ appliqué pendant la fécondation ou au cours des premiers stades du développement embryonnaire. Reprod. Nut. Dev., 26, 1319-1333.

HE X., SCHEURELL M.D., SORANNO P.A., WRIGHT R.A., 1994. Recurrent response patterns of a zooplankton community to whole-lake fish manipulation. Fresh. Biol., $32,61-72$.

JOHANSSON N., KIHLSTRÖM J.E., 1975. Pikes (Esox lucius L.) show to be affected by low $\mathrm{pH}$ values during first weeks after hatching. Env. Research, 9, 12-17.

KWAIN W., ROSE G.A., 1985. Growth of brook trout Salvelinus fontinalis subject to sudden reductions of $\mathrm{pH}$ during their early life history. Trans. Amer. Fish. Soc., 114, 564-570.

LEE R.M., GERKING S.D., 1980. Sensitivity of fish eggs to acid stress. Water Res., 14, 1679-1681.

LEIVESTAD H., MUNIZ I.P., 1976. Fish kill at low pH in a Norwegian river. Nature, 259, 391-392.

LITTLE E.E., FINGER S.E., 1990. Swimming behaviour as an indicator of sublethal toxicity in fish. Environ. Toxicol. Chem., 9, 13-19.

LLOYD R., JORDAN D.H.M., 1964. Some factors affecting the resistance of rainbow trout (Salmo gairdneri) to acid waters. Int. J. Air Water Pollut., 8, 393-403.

Mc WILLIAMS P.G., 1982. The effect of calcium on sodium fluxes in the brown trout, Salmo trutta, in neutral and acid water. J. Exp. Biol., 96, 439-442.

MENENDEZ R., 1976. Chronic effects of reduced pH on brook trout (Salvelinus fontinalis). J. Fish Res. Bd Can., 33, 118-123.

MILBRINK G., JOHANSSON N., 1975. Some effects of acidification on roe of roach, Rutilus rutilus L., and perch, Perca fluviatilis L. Rep. Inst. Freshw. Res. Drottningholm, 54, 52-62.

MOUNT D.I., 1973. Chronic effect of low pH on fathead minnow survival, growth and reproduction. Water Res., 7, 987-993.

RASK M., 1983. The effect of low pH on perch, Perca fluviatilis L. I. : Effects of low pH on the development of eggs of perch. Ann. Zool. Fennici, 20, 73-76.

RASK M., 1984. The effect of low pH on perch, Perca fluviatilis. III. : The perch population in a small, acidic, extremely humic forest lake. Ann. Zool. Fennici, 21, 15-22.

READER J.P., EVERALL N.C., SAYER M.D.J., MORRIS R., 1989. The effects of eight trace metals in acid soft water on survival, mineral uptake and skeletal calcium deposition in yolk-sac fry of brown trout, Salmo trutta L. J. Fish Biol., 35, 187-198.

RUNN P., JOHANSSON N., MILBRINK G., 1977. Some effects of low pH on the hatchability of eggs of perch, Perca fluviatilis. Zoon, 5, 115-125.

RYNANEN R., 1961. Über die Einwirkung von Grubenabfällen auf einen dystrophen See. Ann. Zool. Soc. Vanamo, 22, 161-174.

SAYER M.D.J., READER J.P., DALZIEL T.R.K., 1993. Freshwater acidification : effects on the early life stages of fish. Reviews in Fish Biol. and Fisheries, 3, 95-132.

SCHÄPERCLAUS W., 1962. Traité de pisciculture en étang. 2ème édition française. Vigot Frères, Paris, $620 \mathrm{p}$.

SCHRECK C.B., MOYLE P.B., 1990. Methods for fish biology. Am. Fish. Soc., Bethesda, USA, $684 \mathrm{p}$.

THOMSEN A., KORSGAARD B., JOENSEN J., 1988. Effect of aluminium and calcium ions on survival and physiology of rainbow trout Salmo gairdneri (Richardson) eggs and larvae exposed to acid stress. Aquatic Toxicology, 12, 291-300.

TROJNAR J.R., 1977. Egg hatchability and tolerance of brook trout (Salvelinus fontinalis) fry at low pH. J. Fish. Res. Bd Can., 34, 575-579. 
VALLIN S., 1952. Zwei azidotophe Seen im Küstengebiet vor Nordschurden. Rep. Inst. Freshwater Res. Drottningholm, 34, 167-189.

WEBB D.J., NUCCITELLI R., 1982. Intracellular pH changes accompanying the activation of development in Xenopus eggs : studies with $\mathrm{pH}$ microelectrodes and $31 \mathrm{P}$. MNR. In NUCCITELLI R. and DEAMER D.N., Intracellular pH : Its measurement regulation and utilization in cellular functions, 293-324, A.R. Liss Publ., New York.

WEBB D.J., 1991. A pH sensitive K+ conductance in the egg membrane of the teleost fish Oryzias latipes disappears after fertilization. J. Physiol., 446, $126 p$.

WESTFALL B.A., 1945. Coagulation anoxia in fishes. Ecology, 26, 283-287. 